\title{
Endoscopic Ultrasound-Guided Pancreatic Transmural Stenting and Transmural Intervention
}

\author{
Takeshi Ogura, Hideko Ohama and Kazuhide Higuchi \\ 2nd Department of Internal Medicine, Osaka Medical College, Osaka, Japan
}

Endoscopic ultrasound (EUS)-guided pancreatic access is an emergent method that can be divided into the two main techniques of EUS-guided rendezvous and pancreatic transmural stenting (PTS). While many reports have described EUS-guided procedures, the indications, technical tips, clinical effects, and safety of EUS-guided pancreatic duct drainage (EUS-PD) remain controversial. This review describes the current status of and problems associated with EUS-PD, particularly PTS. We reviewed clinical data derived from a total of 334 patients. Rates of technical and clinical success ranged from $63 \%$ to $100 \%$ and $76 \%$ to $100 \%$, respectively. In contrast, the rate of procedure-related adverse events was high at $26.7 \%$ (89/334). The most frequent adverse events comprised abdominal pain $(n=38)$, acute pancreatitis $(n=15)$, bleeding $(n=9)$, and issues associated with pancreatic juice leakage such as perigastric fluid, pancreatic fluid collection, or pancreatic juice leaks $(n=8)$. In conclusion, indications for EUS-PTS are limited, as is the evidence of its viability, due to the scarcity of expert operators. Despite improvements made to various devices, EUS-PTS remains technically challenging. Therefore, a long-term, large-scale, multicenter study is required to establish this technique as a viable alternative drainage method. Clin Endosc 2020;53:429-435

Key Words: Endoscopic retrograde cholangiopancreatography; Endoscopic ultrasound; Endoscopic ultrasound-guided pancreatic duct drainage; Pancreas; Pancreatic duct stricture

\section{INTRODUCTION}

Pancreatic duct drainage is mainly indicated for pancreatic duct obstruction, stricture, or disruption such as that caused by symptomatic chronic pancreatitis. Most of these conditions can be treated under endoscopic retrograde cholangiopancreatography (ERCP) guidance. However, ERCP fails at times due to various factors, and alternative approaches such as surgery or percutaneous pancreatic duct drainage must be considered. ${ }^{1,2}$ However, surgery is overly invasive for patients

Received: July 2, 2019 Revised: July 26, 2019

Accepted: August 28, 2019

Correspondence: Takeshi Ogura

2nd Department of Internal Medicine, Osaka Medical College, 2-7 Daigakuchou, Takatsukishi, Osaka 569-8686, Japan

Tel: +81-72-683-1221, Fax: +81-72-684-6532, E-mail: oguratakeshi0411@yahoo. co.jp

ORCID: https://orcid.org/0000-0003-2916-6568

(c) This is an Open Access article distributed under the terms of the Creative Commons Attribution Non-Commercial License (http://creativecommons.org/ licenses/by-nc/3.0) which permits unrestricted non-commercial use, distribution, and reproduction in any medium, provided the original work is properly cited. who have other organs that have failed or advanced malignant tumors. Moreover, percutaneous access has several disadvantages such as external drainage, risk of pancreatic juice leakage, and cosmetic issues. Recently, endoscopic ultrasound (EUS)-guided pancreatic access has emerged as an alternative method. Harada et al. reported EUS-guided pancreatography after failed ERCP. ${ }^{3}$ Subsequently, EUS-guided rendezvous (EUS-RV) and pancreatic transmural stenting (PTS) were initially described in 2002. Since then, although many reports have described EUS-guided procedures, the indications, technical tips, clinical effects, and safety of EUS-guided pancreatic duct drainage (EUS-PD) remain controversial.

This review describes the current status of and problems associated with EUS-PD, particularly PTS.

\section{CURRENT INDICATIONS FOR EUS-PD}

EUS-PD is a potential alternative to surgical treatment or ERCP. However, indications for EUS-PD have not been estab- 
lished. Possible indications in terms of technical and anatomical factors include failed pancreatic duct cannulation, inability to pass a guidewire through a pancreatic duct stricture, and inaccessible papilla due to surgically altered anatomy or malignant duodenal obstruction. ${ }^{1-7}$

\section{CLINICAL REVIEW OF PREVIOUS EUS- PTS STUDIES OF AT LEAST 20 PATIENTS}

Table 1 shows the published reports of EUS-PTS that include at least 20 patients. ${ }^{7-12}$ We reviewed clinical data derived from a total of 334 patients. Rates of technical and clinical success ranged from $63 \%$ to $100 \%$ and from $76 \%$ to $100 \%$, respectively. In contrast, the rate of procedure-related adverse events was high at $26.7 \%(89 / 334)$. The most frequent adverse events were abdominal pain $(n=38)$, acute pancreatitis $(n=15)$, bleeding $(n=9)$, and issues associated with pancreatic juice leakage such as perigastric fluid, pancreatic fluid collection, or pancreatic juice leaks $(n=8)$.

\section{TECHNICAL REVIEW OF EUS-PD}

The two types of EUS-PD are EUS-RV and EUS-PTS, including antegrade and retrograde transluminal stenting. To ensure the optimal alignment of the puncture angle and various devices, the main pancreatic duct should be continuously monitored during procedures.

Fig. 1. presents the technical tips for EUS-RV. The main pancreatic duct is punctured using a $19 \mathrm{G}$ needle, and contrast medium is injected for pancreatography (Fig. 1A). A guidewire is then inserted into the intestine across the stricture site (Fig. 1B). After the EUS scope is withdrawn (Fig. 1C), a duodenoscope is inserted into the ampulla of Vater (Fig. 1C) and the guidewire is grasped using devices such as forceps or a basket catheter (Fig. 1D). After the guidewire is pulled into the duodenoscope, the pancreas is cannulated over the guidewire (Fig. 1E).

Fig. 2. presents the technical tips for EUS-PTS using a plastic stent. The main pancreatic duct can be identified from the stomach or duodenal bulb as access routes. The main pancreatic duct is punctured using a $19 \mathrm{G}$ needle. If this is challenging, puncture sites can be accessed from the upper portion of the stomach. Thereafter, an echoendoscope can be inserted

Table 1. Literature Review of Endoscopic Ultrasound-Guided Pancreatic Transmural Stenting including at Least 20 Cases

\begin{tabular}{|c|c|c|c|c|c|c|c|c|}
\hline Study & $\begin{array}{l}\text { Patients } \\
\text { (n) }\end{array}$ & Access route & $\begin{array}{l}\text { Puncture } \\
\text { needle } \\
\text { (G) }\end{array}$ & Dilation device & Kinds of stent & $\begin{array}{l}\text { Technical } \\
\text { success }\end{array}$ & Adverse events ( $n$ ) & $\begin{array}{l}\text { Clinical } \\
\text { success }\end{array}$ \\
\hline $\begin{array}{l}\text { Tessier et al. } \\
(2007)^{7}\end{array}$ & 36 & $\begin{array}{l}\text { Stomach (29) } \\
\text { Bulb (7) }\end{array}$ & $\begin{array}{l}19 \mathrm{G}, \\
22 \mathrm{G}\end{array}$ & Diathermic dilator & 6 or 7 Fr PS & $92 \%$ & $\begin{array}{l}\text { Severe: hematoma (1), AP with } \\
\text { PS (1) Mild: N/D (2) }\end{array}$ & $76 \%$ \\
\hline $\begin{array}{l}\text { Fujii et al. } \\
\quad(2013)^{8}\end{array}$ & 43 & $\mathrm{~N} / \mathrm{D}$ & $19 \mathrm{G}$ & $\begin{array}{l}\text { Balloon, tapered } \\
\text { catheters, nee- } \\
\text { dle-knife }\end{array}$ & Pig or straight PS & $74 \%$ & $\begin{array}{l}\text { Abdominal pain (13), abscess } \\
\text { (1), guidewire shaving (1), } \\
\text { AP (1) }\end{array}$ & $93 \%$ \\
\hline $\begin{array}{l}\text { Will et al. } \\
(2015)^{10}\end{array}$ & 83 & $\mathrm{~N} / \mathrm{D}$ & $19 \mathrm{G}$ & Balloon, ring-knife & $\begin{array}{l}\text { Pig or straight PS } \\
\text { (5-10 Fr), covered } \\
\text { SEMS, LAMS }\end{array}$ & $63 \%$ & $\begin{array}{l}\text { AP (6), bleeding (6), abscess } \\
\text { (6), perigastric fluid (3), ulcer } \\
\text { (2), aspiration (1), perfora- } \\
\text { tion (1), retention cyst (1) }\end{array}$ & $82 \%$ \\
\hline $\begin{array}{l}\text { Oh et al. } \\
(2016)^{11}\end{array}$ & 25 & $\begin{array}{l}\text { Stomach (23) } \\
\text { Bulb (1) } \\
\text { Jejunum (1) }\end{array}$ & $19 \mathrm{G}$ & $\begin{array}{l}\text { Balloon, } \\
\text { needle-knife }\end{array}$ & $\begin{array}{l}\text { Modified covered } \\
\text { SEMS }\end{array}$ & $100 \%$ & $\begin{array}{l}\text { Abdominal pain (4), bleeding } \\
\text { (1) }\end{array}$ & $100 \%$ \\
\hline $\begin{array}{l}\text { Tyberg et al. } \\
(2017)^{12}\end{array}$ & 80 & $\mathrm{~N} / \mathrm{D}$ & $19 \mathrm{G}$ & Cautery, balloon & Pig PS (5-10 Fr) & $89 \%$ & $\begin{array}{l}\text { AP (6), pancreatic fluid collec- } \\
\text { tion (4), abdominal pain (3), } \\
\text { bleeding (1), MPD leak (1), } \\
\text { perforation (1) }\end{array}$ & $92 \%$ \\
\hline $\begin{array}{l}\text { Chen et al. } \\
(2017)^{13}\end{array}$ & 37 & N/D & $\begin{array}{l}19 \mathrm{G} \\
22 \mathrm{G}\end{array}$ & $\mathrm{N} / \mathrm{D}$ & PS & $92 \%$ & $\begin{array}{l}\text { Abdominal pain (13), abscess } \\
\text { (1), ulcer (1) }\end{array}$ & $85 \%$ \\
\hline $\begin{array}{l}\text { Matsunami et } \\
\text { al. }(2018)^{14}\end{array}$ & 30 & N/D & $\begin{array}{l}19 \mathrm{G}, \\
22 \mathrm{G}\end{array}$ & $\begin{array}{l}\text { Balloon, electro- } \\
\text { cautery dilator, } \\
\text { mechanical dilator }\end{array}$ & Dedicated PS (7 Fr) & $100 \%$ & $\begin{array}{l}\text { Abdominal pain (5), AP (1), } \\
\text { bleeding (1) }\end{array}$ & $100 \%$ \\
\hline
\end{tabular}

AP, acute pancreatitis; LAMS, lumen apposing metal stent; MPD, main pancreatic duct; N/D, not discussed; PS, plastic stent; SEMS, self-expandable metal stent. 
into the second part of the duodenum and pulled from the stomach while visualizing the main pancreatic duct. In this manner, the main pancreatic duct can be punctured from around the lower or middle portions of the stomach, which should be easier (Fig. 2A, B). Having punctured the main pancreatic duct, contrast medium is injected and a guidewire is inserted into the main pancreatic duct. A fistula can be dilated using a balloon catheter or mechanical or electrocautery dilators (Fig. 2C). Finally, a plastic stent is deployed from the main pancreatic duct to the stomach (Fig. 2D).

\section{Which site should be punctured?}

The main pancreatic duct can be identified from the du- odenum or stomach. At present, duodenal or gastric access routes of transluminal stenting seem equally effective from the technical and clinical perspectives. Most published reports describe the transgastric approach. Tessier et al. noted that the transduodenal approach may be preferable because the "long" scope position allows a better view of the main pancreatic duct, better stability during the procedure, and better stent "pushability.".7.7 However, if a stricture is located in the pancreatic body, the transduodenal approach might be complicated in terms of passing a guidewire through the stricture site. In addition, the gastroduodenal artery is sometimes visible on EUS images while locating the main pancreatic duct from the duodenal bulb. Therefore, this access route may be associat-
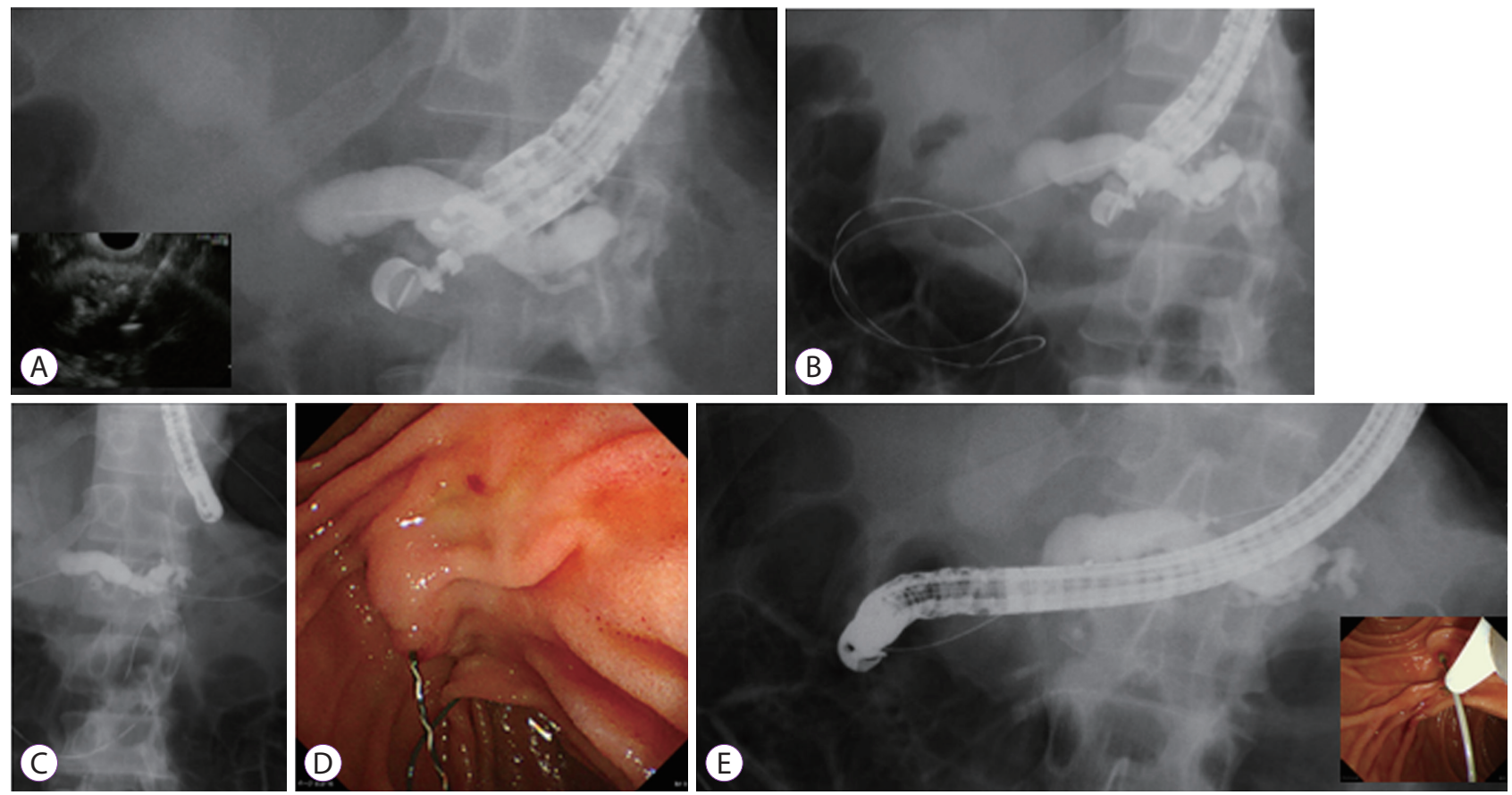

Fig. 1. Technique of endoscopic ultrasound-guided rendezvous. (A) The main pancreatic duct is punctured using $19 \mathrm{G}$ needle, and the contrast medium is injected. (B) The guidewire is inserted into the intestine across the stricture site. (C) The echoendoscope is withdrawn. (D) A duodenoscope is inserted into the ampulla of Vater. (E) After the guidewire is pulled into a duodenoscope, pancreatic cannulation is performed over the guidewire (E).
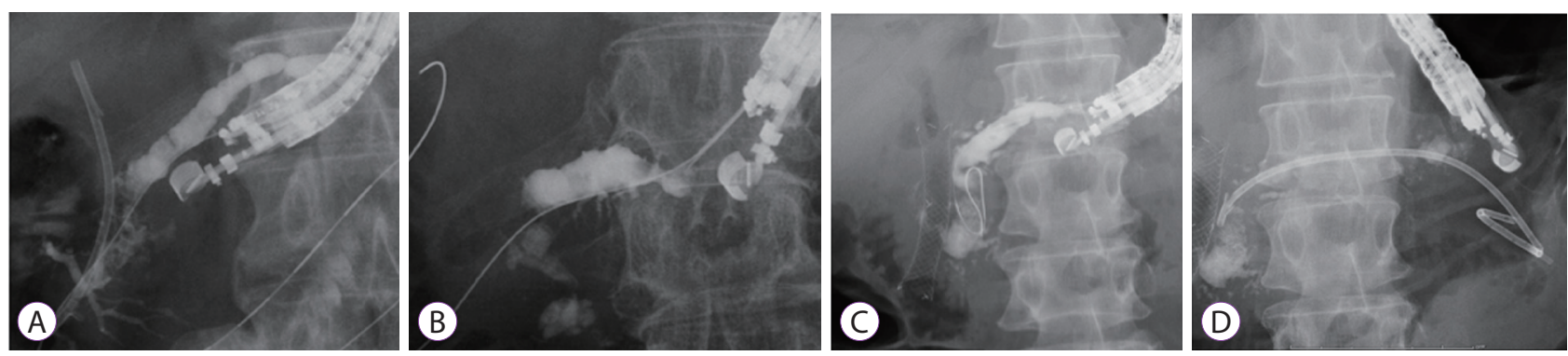

Fig. 2. Technical tips for endoscopic ultrasound-guided pancreatic transluminal stenting. (A) The main pancreatic duct is punctured from the lower or middle of the stomach. (B) The main pancreatic duct is punctured from the upper site of the stomach. (C) The guidewire is inserted into the main pancreatic duct, and the fistula is dilated using a balloon catheter. (D) Plastic stent deployment from the main pancreatic duct to the stomach is performed. 
ed with bleeding due to the burning effects of dilation using electrocautery. We concluded that the puncture site should be determined according to the ease of puncturing, the reason for the procedure, and the status of the patient including the stricture site(s).

Almost all authors have selected a $19 \mathrm{G}$ needle for fine needle aspiration. After the main pancreatic duct is punctured, tract dilation is needed to insert a stent delivery system, preferably using a stiff 0.025 or 0.035 inch guidewire, which can be inserted into a $19 \mathrm{G}$ needle. However, when the pancreatic parenchyma is fibrotic, which occurs in patients with chronic pancreatitis, the puncturing itself can pose a challenge. In this situation, a $22 \mathrm{G}$ needle can be applied to enable the use of a 0.018 or 0.021 inch guidewire. A novel electrocautery, balloon, and mechanical dilator has recently entered the market, ${ }^{13-15}$ which has facilitated tract dilation under thin guidewire deployment.

\section{Which dilation devices should be used?}

During EUS-PTS, a stent delivery system might need to be inserted via the luminal wall, pancreatic parenchyma, and pancreatic duct. These tips for tract dilation are quite similar to those for EUS-guided biliary drainage. However, tract dilation can be complicated by a fibrotic pancreatic parenchyma during EUS-PTS. Various dilation devices such as mechanical, balloon, or electrocautery dilators are now available. From the viewpoint of adverse events, the risks associated with non-electrocautery-assisted devices include pancreatic leaks, perforation, and bleeding due to radial or axial dilation force ${ }^{16}$ Indeed, Honjo et al. described the risk of bleeding due to this type of device ${ }^{14}$ among 64 patients who underwent EUS-hepaticogastrostomy $(n=49)$ and EUS-PD $(n=15))^{16}$ They evaluated the technical and clinical success and adverse event rates between groups in which the dilation was mechanical or performed via electrocautery. Neither the procedural duration (28.4 $\pm 19.6 \mathrm{~min}$ vs. $27.2 \pm 7.7 \mathrm{~min}, p=0.58$ ) nor the technical success rates of tract dilation ( $9 / 10$ vs. $5 / 5, p=0.71)$ significantly differed. The adverse event of bleeding occurred only in the electrocautery group, but the difference did not reach significance. Therefore, Honjo et al. concluded that an ultra-tapered mechanical dilator is useful because it reduces postprocedural bleeding with a higher technical success rate than that of the electrocautery dilator. ${ }^{16}$ Tract dilation using non-cautery-assisted devices can be initially attempted for normal pancreatic parenchyma to avoid injury induced by cautery. ${ }^{17,18}$

However, if tract dilation in patients with chronic pancreatitis is challenging, electrocautery dilation might be necessary despite the risk of bleeding due to burning. In this situation, an electrocautery dilator that is coaxial with the guidewire should be used, though adverse events due to burning can still arise.

A novel, fine-gauge electrocautery dilator to reduce burning effects (Fine 025; Medico's HIRATA Inc., Osaka, Japan) is now available in Japan. ${ }^{13}$ The distal end of the outer dilator contains a metal tip, the top of which is only $3 \mathrm{Fr}$. This electrocautery dilator is wire-guided and coaxial with a 0.025 inch guidewire. Although technical feasibility and safety should be evaluated in a prospective trial, this dilator may be useful for EUS-PTS. Finally, the most appropriate dilation device should be evaluated in a randomized, controlled trial.

\section{Which drainage devices should be used?}

A prospective comparison of stent types has not been implemented. Published reports indicate that plastic stents including straight and double pigtail types are the most frequently deployed. Compared with EUS-hepaticogastrostomy, stents can migrate into the abdominal cavity because the pancreatic parenchyma and stomach wall are situated close to each other. In addition, the pancreatic duct does not dilate very much at times, in which case a plastic stent might be suitable. Matsunami et al. described EUS-PTS using a new, 7 Fr single pigtail plastic stent with a total length of $20 \mathrm{~cm}$ and an effective length of $15 \mathrm{~cm}$ in 30 patients with acute recurrent pancreatitis caused by stricture of the main pancreatic duct or stenotic pancreatojejunostomy. ${ }^{12,14}$ Technical success was achieved in all patients within $30.8 \pm 13.5 \mathrm{~min}$. Adverse events included abdominal pain or bleeding, but none of the stents migrated in any of the patients.

Fully covered self-expandable covered metal stents (FCSEMS) offer several advantages for EUS-PTS but also raise concerns. Compared with plastic stents, the large diameter of FCSEMS might facilitate effective drainage and easy re-intervention. If tract dilation is attempted using an electrocautery dilator, bleeding risk may be reduced due to the tamponade effect of FCSEMS. Moreover, these stents might remain patent for longer periods than plastic stents, and pancreatic juice leaks might be prevented by the tamponade effect. The downsides are that ductal change may be induced due to the gap between the FCSEMS and the main pancreatic duct. ${ }^{19}$ In addition, pancreatic duct obstruction can occur via crossstream blockage of the main pancreatic duct with the covering membrane of an FCSEMS. However, this disadvantage has been described. ${ }^{8}$ The study of Oh et al. included 25 consecutive patients with painful obstructive pancreatitis who underwent EUS-PTS with a novel FCSEMS after failed ERCP. ${ }^{11}$ The anchoring flaps of the novel FCSEMS were designed with a blunt end covered by a membrane to prevent stent-induced ductal injury and proximal and distal migration. Tract dilation was firstly attempted using a needle-knife, followed by a 4-mm balloon catheter to insert the novel FCSEMS. The tech- 
nical success rate was $100 \%$ (25/25), and the median procedure duration was $25 \mathrm{~min}$ (interquartile range, 14-42.5). Although adverse events developed in five patients (25\%; self-limiting abdominal pain $[n=4]$, minor bleeding $[n=1])$, stent-related adverse events such as pneumoperitoneum, stent migration, stent clogging, stent-related pancreatitis, and pancreatic sepsis did not arise in any patients during a follow-up period of $221.1 \pm 190.48$ days. In addition, definite or aggravated upstream ductal dilation by cross-stream obstruction of the main pancreatic duct that covers the membrane of the FCSEMS was not evident on follow-up computed tomography images. Therefore, according to this study, FCSEMS might be safe and feasible, although a prospective randomized trial is needed to compare EUS-PTS with plastic stents and FCSEMS.

A lumen-apposing metal stent (LAMS) has been developed for EUS-guided transluminal intervention, including pancreatic fluid collection and drainage, ${ }^{20}$ biliary drainage, ${ }^{21}$ and gastrojejunostomy. ${ }^{22}$ The unique design, in terms of anchoring flanges, may play an important role in preventing stent migration. However, compared with other organs, the pancreatic duct is not usually highly dilated, particularly when it is ob- structed. Since the lumen of the main pancreatic duct is narrower, indications for LAMS might be limited for EUS-PTS.

\section{LONG-TERM OUTCOMES AFTER EUS- PTS}

Most published reports of EUS-PTS have focused on the main outcomes of technical success and short-term follow-up results. As a result, the long-term outcomes are unclear. Matsunami et al. evaluated the outcomes of EUS-PTS using dedicated plastic stents in 25 patients who were clinically followed up for a median of 23 (range, 6-44) months..$^{10,14}$ Among them, 20 patients required an average of 3 (range, 1-12) scheduled stent exchanges. Four patients desired to undergo a repeat stent exchange after the initial EUS-PTS. Spontaneous stent dislocation was a late adverse event in $6(24 \%)$ patients, and 12 patients underwent scheduled stent exchange 1 year after the initial EUS-PTS. Three patients were successfully converted to a standard transpapillary pancreatic duct stent under ERCP, and 9 (36\%) patients had complete stent re-
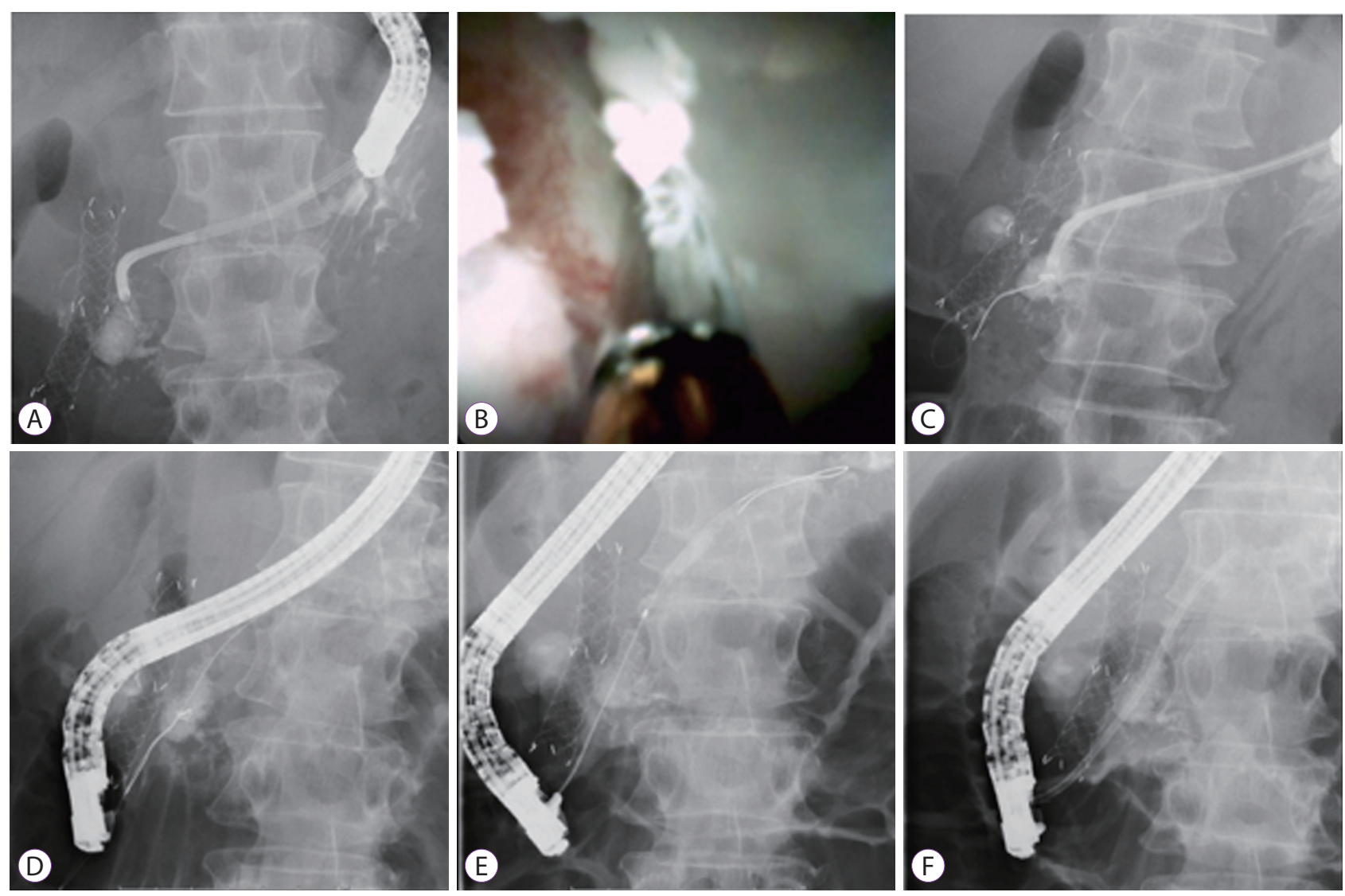

Fig. 3. Endoscopic ultrasound-guided antegrade intervention. (A) A pancreatoscope is inserted into the main pancreatic duct. (B) Antegrade endoscopic hydraulic lithotripsy is attempted. (C) The guidewire is successfully inserted into the intestine across huge pancreatic stones. (D) The guidewire is grasped using a basket catheter. (E) Pancreatic duct cannulation is performed. (F) Plastic stent deployment is performed. 
moval, either intentionally or by asymptomatic spontaneous dislodgement. The clinical success rate was $92 \%$ (23/25), and pancreatitis recurred in 2 (8\%) of 25 patients. In addition, none of the patients needed surgical repair. Therefore, they concluded that EUS-PTS using dedicated plastic stents was associated with long-term clinical success for most patients. According to Matsunami et al., EUS-PTS might be promising for both short- and long-term outcomes. ${ }^{14}$ However, a prospective multicenter study with a longer clinical follow-up is warranted to confirm this result.

\section{TRANSLUMINAL INTERVENTION THROUGH THE EUS-PTS ROUTE}

Various interventions are possible after creating a fistula between the main pancreatic duct and the stomach. ${ }^{23-25}$ Fig. 3 shows technical tips for EUS-RV assisted by antegrade endoscopic hydraulic lithotrispy. The stent is removed after EUSPTS, and then the fistula is dilated using a balloon catheter to insert a SpyScope ${ }^{\mathrm{TM}}$ DS pancreatoscope (Boston Scientific, Marlborough, MA, USA). The pancreatoscope is inserted into the main pancreatic duct (Fig. 3A), and a pancreatic stone is identified. Antegrade endoscopic hydraulic lithotrispy is attempted (Fig. 3B). The stone is fragmented, and the guidewire is advanced into the intestine across the stone (Fig. 3C). The guidewire is grasped using a basket catheter (Fig. 3D), and the pancreatic duct is cannulated (Fig. 3E). Finally, a plastic stent is deployed (Fig. 3F). In our experience, a fistula is created after 1 week, but this procedure is quite complex. Therefore, we recommend performing this procedure 1 month after EUSPTS.

\section{CONCLUSIONS}

Indications for EUS-PTS are limited, as is the evidence of its viability, due to the scarcity of expert operators. Despite improvements made to various devices, EUS-PTS remains technically challenging. Therefore, a long-term, large-scale, multicenter study is required to establish this technique as a viable alternative drainage method.

Conflicts of Interest

The authors have no financial conflicts of interest.

\section{Acknowledgments}

I am grateful to all the members of the International Digestive Endoscopy Network (IDEN 2019). I especially thank my Korean colleagues with whom I participated in several valuable discussions.
ORCID

Hideko Ohama: https://orcid.org/0000-0002-1882-824X

Kazuhide Higuchi: https://orcid.org/0000-0002-4142-2272

\section{REFERENCES}

1. Ahmed Ali U, Pahlplatz JM, Nealon WH, van Goor H, Gooszen HG, Boermeester MA. Endoscopic or surgical intervention for painful obstructive chronic pancreatitis. Cochrane Database Syst Rev 2015;(3):CD007884

2. Mizandari M, Azrumelashvili T, Kumar J, Habib N. Percutaneous image-guided pancreatic duct drainage: technique, results and expected benefits. Cardiovasc Intervent Radiol 2017;40:1911-1920.

3. Harada N, Kouzu T, Arima M, Asano T, Kikuchi T, Isono K. Endoscopic ultrasound-guided pancreatography: a case report. Endoscopy 1995;27:612-615.

4. Bataille L, Deprez P. A new application for therapeutic EUS: main pancreatic duct drainage with a "pancreatic rendezvous technique". Gastrointest Endosc 2002;55:740-743.

5. François E, Kahaleh M, Giovannini M, Matos C, Devière J. EUS-guided pancreaticogastrostomy. Gastrointest Endosc 2002;56:128-133.

6. Kahaleh M, Yoshida C, Yeaton P. EUS antegrade pancreatography with gastropancreatic duct stent placement: review of two cases. Gastrointest Endosc 2003;58:919-923.

7. Tessier G, Bories E, Arvanitakis M, et al. EUS-guided pancreatogastrostomy and pancreatobulbostomy for the treatment of pain in patients with pancreatic ductal dilatation inaccessible for transpapillary endoscopic therapy. Gastrointest Endosc 2007;65:233-241.

8. Fujii LL, Topazian MD, Abu Dayyeh BK, et al. EUS-guided pancreatic duct intervention: outcomes of a single tertiary-care referral center experience. Gastrointest Endosc 2013;78:854-864.e851.

9. Itoi T, Kasuya K, Sofuni A, et al. Endoscopic ultrasonography-guided pancreatic duct access: techniques and literature review of pancreatography, transmural drainage and rendezvous techniques. Dig Endosc 2013;25:241-252.

10. Will U, Reichel A, Fueldner F, Meyer F. Endoscopic ultrasonography-guided drainage for patients with symptomatic obstruction and enlargement of the pancreatic duct. World J Gastroenterol 2015;21:1314013151.

11. Oh D, Park DH, Cho MK, et al. Feasibility and safety of a fully covered self-expandable metal stent with antimigration properties for EUS-guided pancreatic duct drainage: early and midterm outcomes (with video). Gastrointest Endosc 2016;83:366-373.e2.

12. Tyberg A, Sharaiha RZ, Kedia P, et al. EUS-guided pancreatic drainage for pancreatic strictures after failed ERCP: a multicenter international collaborative study. Gastrointest Endosc 2017;85:164-169.

13. Chen YI, Levy MJ, Moreels TG, et al. An international multicenter study comparing EUS-guided pancreatic duct drainage with enteroscopy-assisted endoscopic retrograde pancreatography after Whipple surgery. Gastrointest Endosc 2017;85:170-177.

14. Matsunami Y, Itoi T, Sofuni A, et al. Evaluation of a new stent for EUS-guided pancreatic duct drainage: long-term follow-up outcome. Endosc Int Open 2018;6:E505-E512.

15. Ogura T, Nakai Y, Itoi T. Novel fine gauge electrocautery dilator for endoscopic ultrasound-guided hepaticogastrostomy (with video). J Hepatobiliary Pancreat Sci 2019;26:E3-E4.

16. Honjo M, Itoi T, Tsuchiya T, et al. Safety and efficacy of ultra-tapered mechanical dilator for EUS-guided hepaticogastrostomy and pancreatic duct drainage compared with electrocautery dilator (with video). Endosc Ultrasound 2018;7:376-382.

17. Amano M, Ogura T, Onda S, et al. Prospective clinical study of endoscopic ultrasound-guided biliary drainage using novel balloon catheter 
(with video). J Gastroenterol Hepatol 2017;32:716-720.

18. Fujii-Lau LL, Levy MJ. Endoscopic ultrasound-guided pancreatic duct drainage. J Hepatobiliary Pancreat Sci 2015;22:51-57.

19. Krafft MR, Nasr JY. Anterograde endoscopic ultrasound-guided pancreatic duct drainage: a technical review. Dig Dis Sci 2019;64:1770-1781.

20. Nakai Y, Kogure H, Isayama H, Koike K. Endoscopic ultrasound-guided pancreatic duct drainage. Saudi J Gastroenterol 2019;25:210-217.

21. Moon SH, Kim MH, Park DH, et al. Modified fully covered self-expandable metal stents with antimigration features for benign pancreatic-duct strictures in advanced chronic pancreatitis, with a focus on the safety profile and reducing migration. Gastrointest Endosc 2010;72:8691.

22. Chen YI, Barkun AN, Adam V, et al. Cost-effectiveness analysis com- paring lumen-apposing metal stents with plastic stents in the management of pancreatic walled-off necrosis. Gastrointest Endosc 2018;88:267276.el.

23. Kunda R, Pérez-Miranda M, Will U, et al. EUS-guided choledochoduodenostomy for malignant distal biliary obstruction using a lumen-apposing fully covered metal stent after failed ERCP. Surg Endosc 2016;30:5002-5008.

24. Itoi T, Baron $\mathrm{TH}$, Khashab MA, et al. Technical review of endoscopic ultrasonography-guided gastroenterostomy in 2017. Dig Endosc 2017;29:495-502.

25. James TW, Baron TH. Antegrade pancreatoscopy via EUS-guided pancreaticogastrostomy allows removal of obstructive pancreatic duct stones. Endosc Int Open 2018;6:E735-E738. 